\title{
Divergent Longitudinal Propagation of White Matter Degradation in Logopenic and Semantic Variants of Primary Progressive Aphasia
}

\author{
Sicong Tu ${ }^{\mathrm{a}, \mathrm{b}, \mathrm{c}}$, Cristian E. Leyton ${ }^{\mathrm{a}, \mathrm{b}, \mathrm{d}}$, John R. Hodges ${ }^{\mathrm{a}, \mathrm{b}, \mathrm{c}}$, Olivier Piguet ${ }^{\mathrm{a}, \mathrm{b}, \mathrm{c}}$ \\ and Michael Hornberger ${ }^{\mathrm{b}, \mathrm{c}, \mathrm{e}, *}$ \\ ${ }^{a}$ Neuroscience Research Australia, Randwick, Sydney, Australia \\ ${ }^{\mathrm{b}}$ Australian Research Council Centre of Excellence in Cognition and its Disorders, Sydney, Australia \\ ${ }^{\mathrm{c}}$ School of Medical Sciences, University of New South Wales, Sydney, Australia \\ ${ }^{\mathrm{d}}$ Faculty of Health Sciences, University of Sydney, Sydney, Australia \\ ${ }^{\mathrm{e}}$ Department of Clinical Neurosciences, University of Cambridge, Cambridge, UK
}

Handling Associate Editor: Sharon Naismith

Accepted 15 September 2015

\begin{abstract}
.
Background: Clinico-pathological distinction of primary progressive aphasia (PPA) can be challenging at clinic presentation. In particular, cross-sectional neuroimaging signatures across the logopenic (lvPPA) and semantic (svPPA) variants are difficult to establish, with longitudinal profiles showing greater divergence.

Objective: Assess longitudinal propagation of white matter degradation in lvPPA and svPPA to determine disease progression over time, and whether this reflects distinct underlying pathology.

Method: A cohort of 27 patients with dementia (12 lvPPA; 15 svPPA) and 12 healthy controls were assessed at baseline and 1-year follow-up on the Addenbrooke's Cognitive Examination-Revised and Sydney Language Battery. Diffusion weighted images were collected at both time-points and analyzed for longitudinal white matter change using DTI-TK and TBSS.

Results: LvPPA patients showed a significant decline in naming and repetition, over 1 year, while svPPA patients declined in naming and comprehension. Longitudinal imaging revealed widespread bilateral degradation of white matter tracts in lvPPA over a 1-year period with early involvement of the left posterior inferior longitudinal fasciculus (ILF). SvPPA demonstrated focal left lateralized white matter degradation involving the uncinate fasciculus (UF) and anterior ILF, propagating to the right UF with disease progression.

Conclusions: LvPPA and svPPA cohorts showed distinct longitudinal cognitive and white matter profiles. We propose differences in multi-centric and focal white matter dysfunction in lvPPA and svPPA, respectively, reflect underlying pathological differences. The clinical relevance of white matter degradation and mechanisms underlying disease propagation are discussed.
\end{abstract}

Keywords: Diffusion tensor imaging, frontotemporal dementia, primary progressive aphasia, white matter

\section{INTRODUCTION}

Primary progressive aphasias (PPA) are a clinicopathological heterogeneous group of neurodegenerative conditions characterized by the progressive breakdown of language skills and associated brain networks [1]. PPA comprises three distinct clinical

\footnotetext{
*Correspondence to: Dr. Michael Hornberger, Department of Clinical Neurosciences, University of Cambridge, Cambridge CB2 0SZ, UK. Tel.: +44 1223 760694; E-mail: mh486@medschl. cam.ac.uk.
} 
syndromes: logopenic (lvPPA), semantic (svPPA), and nonfluent (nfvPPA) variants [1, 2]. Clinical classification into PPA variants occurs at three levels: an initial clinical diagnosis determined by neuropsychological testing, imaging supported structural change, and finally pathological diagnosis either at postmortem or via in vivo PET ligands. Cognitively, lvPPA, svPPA, and nfvPPA variants are characterized by core language deficits involving repetition, comprehension, and agrammatism, respectively [1]. Structural neuroimaging studies also highlight distinct, primarily left hemispheric, patterns of atrophy in temporal and frontal lobe structures, across PPA variants [1-6].

Pathological diagnosis across all PPAs is less clear with the presence of tau and TAR-DNAbinding protein 43 (TDP-43) pathological subtypes of frontotemporal lobar degeneration (FTLD) [7], and Alzheimer's disease (AD) pathology reported in each variant [8-10]. Of all PPA subtypes, lvPPA and svPPA appear to show the most homogenous clinico-pathological mapping, attributed to either AD or FTLD-TDP, respectively [8]. The impact of this distinction in pathology can be clearly seen in the localization and degree of structural abnormality in lvPPA and svPPA. Notably, lvPPA show widespread cortical atrophy to posterior regions of the brain, predominantly affecting the left temporo-parietal junction region, while svPPA show focal change associated with the anterior temporal lobes [2, 3]. However, crosssectional studies comparing white matter integrity in lvPPA and svPPA cohorts often fail to show such a distinction $[11,12]$, likely due to wide distributed white matter changes seen in lvPPA.

The current study investigates whether longitudinal white matter assessment allows a better distinction of clinical lvPPA and svPPA. Importantly, a subgroup of patients had confirmed amyloid in vivo biomarkers to allow pathological confirmation. We hypothesized that differences in disease pathology can be detected using a longitudinal analysis approach, and, in particular, patients with lvPPA will show white matter changes across multiple white matter tracts, relative to svPPA, which will show highly localized white matter changes in the temporal lobes only.

\section{METHOD}

\section{Participants}

Twenty-seven dementia patients (15 svPPA; 12 lvPPA) and 12 healthy controls were recruited from the Sydney frontotemporal dementia research group
(FRONTIER) clinical database. All participants underwent an initial assessment and a 12 \pm 2 -month follow-up assessment. At each visit, patients underwent a comprehensive assessment, which included a clinical interview, neurological examination, cognitive assessment, and whole-brain MRI. All patients met current clinical diagnostic criteria for probable lvPPA and svPPA [1], and diagnosis was established by consensus among the neurologist, neuropsychologist, and occupational therapist. All participants' scans were visually inspected to rule out abnormalities other than atrophy. Participants showing evidence of cerebrovascular disease, including marked small vessel disease, were excluded. Amyloid- $\beta$ deposition using Pittsburgh compound B (PiB) PET was assessed in a subset of PPA patients (9 lvPPA, 6 svPPA). All lvPPA patients scanned were found to be PiB-positive, while svPPA were all negative with the exception of 1 case, which was excluded from analyses. Patient cohorts were matched for age, education, disease duration, and functional disease severity using the Frontotemporal Dementia Rating Scale (FRS) [13] (Table 1). Patients with a change in diagnosis, atypical features, or absence of progressive language impairment were excluded from the study. Healthy controls were matched for education and all scored above 88/100 on the Addenbrooke's Cognitive Examination-Revised (ACE-R) screening of general cognition [14]. Wholebrain MRI scans were visually assessed for structural and white matter abnormalities; none were reported in this group.

\section{Cognitive assessment}

All participants were administered the ACE-R as a general screening of cognition assessing: attention, verbal fluency, visuospatial ability, memory, and language [14]. Patients' language and speech impairment were comprehensively examined using the Sydney Language Battery (SYD-BAT) [15]. The SYD-BAT consists of 30 test words, each representing an imageable noun, 3 or more syllables in length. Each word was assessed for comprehension, naming, repetition, and semantic association, to provide a comprehensive assessment of language and speech impairment. Performance on the ACE-R and SYD-BAT were determined at initial and follow-up assessments.

\section{Imaging acquisition}

All participants underwent whole-brain $T_{1}$ and serial diffusion weighted imaging using a $3 \mathrm{~T}$ Philips 
Table 1

Participant demographic characteristics, global cognition and single-word task performance. Mean and standard deviation reported

\begin{tabular}{|c|c|c|c|c|c|}
\hline & $\begin{array}{l}\text { SvPPA } \\
(n=15)\end{array}$ & $\begin{array}{l}\text { LvPPA } \\
(n=12)\end{array}$ & $\begin{array}{c}\text { Controls } \\
(n=12)\end{array}$ & $\begin{array}{l}\text { Group } \\
\text { Effect }\end{array}$ & $\begin{array}{c}\text { svPPA versus } \\
\text { lvPPA }\end{array}$ \\
\hline Gender (M/F) & $10 \mathrm{M}, 5 \mathrm{~F}$ & $3 \mathrm{M}, 9 \mathrm{~F}$ & $6 \mathrm{M}, 6 \mathrm{~F}$ & - & - \\
\hline Handedness (L/R) & $1 \mathrm{~L}, 14 \mathrm{R}$ & $1 \mathrm{~L}, 11 \mathrm{R}$ & $12 \mathrm{R}$ & - & - \\
\hline Age (y) & $63.4(7.2)$ & $64.4(7.3)$ & $69.7(5.7)$ & $*$ & $\mathrm{n} / \mathrm{s}$ \\
\hline Education $(\mathrm{y})$ & $13.3(3)$ & $12.9(3.8)$ & $13.5(3.3)$ & $\mathrm{n} / \mathrm{s}$ & $\mathrm{n} / \mathrm{s}$ \\
\hline Disease Duration (y) & $4.1(3.3)$ & $4.1(1.4)$ & - & - & $\mathrm{n} / \mathrm{s}$ \\
\hline FRS & $1.3(1.4)$ & $1.3(1.2)$ & - & - & $\mathrm{n} / \mathrm{s}$ \\
\hline \multicolumn{6}{|l|}{ ACE-R: } \\
\hline Total (/100) & $63.3(15.9)$ & $58.9(10.2)$ & $93(4.4)$ & $* *$ & $\mathrm{n} / \mathrm{s}$ \\
\hline \multicolumn{6}{|l|}{ ACE-R (1yr): } \\
\hline Total (/100) & $57.5(16.8)^{\mathrm{a}}$ & $47.5(16.8)^{\mathrm{a}}$ & $93.8(4.4)$ & $* *$ & $\mathrm{n} / \mathrm{s}$ \\
\hline \multicolumn{6}{|l|}{ SYD-BAT: } \\
\hline Comprehension (/30) & $21.3(6.7)^{\dagger}$ & $25.7(2.1)^{\dagger}$ & - & - & $\mathrm{n} / \mathrm{s}$ \\
\hline Naming (/30) & $7.3(5.1)^{\dagger}$ & $13.8(7.8)^{\dagger}$ & - & - & $*$ \\
\hline Repetition (/30) & $28.9(1.9)$ & $23.8(7.2)^{\dagger}$ & - & - & $*$ \\
\hline Semantic Association (/30) & $18.3(6.5)^{\dagger}$ & $24.5(3.4)^{\dagger}$ & - & - & $* *$ \\
\hline \multicolumn{6}{|l|}{ SYD-BAT (1yr): } \\
\hline Comprehension (/30) & $18.8(7)^{\dagger, \mathrm{a}}$ & $24.9(3.2)^{\dagger}$ & - & - & * \\
\hline Naming (/30) & $6.6(5.2)^{\dagger, \mathrm{a}}$ & $10.5(6.9)^{\dagger, \mathrm{a}}$ & - & - & $\mathrm{n} / \mathrm{s}$ \\
\hline Repetition (/30) & $29(1.2)$ & $19.5(9.6)^{\dagger, \mathrm{a}}$ & - & - & $*$ \\
\hline Semantic Association (/30) & $17.2(6.6)^{\dagger, \mathrm{a}}$ & $23.8(4)^{\dagger}$ & - & - & $* *$ \\
\hline
\end{tabular}

FRS, Frontotemporal dementia rating scale Rasch score; ACE-R, Addenbrooke's cognitive examination-Revised; SYD-BAT, Sydney language battery. Behavioral data on the ACE-R (1 y) was available for 10 Controls; SYD-BAT was not administered to Controls. Normative scores for the SYD-BAT: Comprehension=29.1 (1.1); Naming= 26.6 (2.1); Repetition = 29.9 (0.3); Semantic Association= 27.7 (1.4). ${ }^{\dagger}$ Denotes SYD-BAT performance 2 S.D below mean Control normative score. $\mathrm{n} / \mathrm{s}$, not significant; ${ }^{*} p<0.05 ;{ }^{* *} p<0.001$; ${ }^{\text {a }}$ significant change from baseline $(p<0.05)$

MRI scanner with standard quadrature head coil (eight channels) at initial and follow-up assessments. Structural $\mathrm{T}_{1}$-weighted images were acquired using the following sequences: coronal orientation, matrix $256 \times 256,200$ slices, $1 \times 1 \mathrm{~mm}$ in-plane resolution, slice thickness $1 \mathrm{~mm}$, echo time/repetition time = $2.6 / 5.8 \mathrm{~ms}$, flip angle $\alpha=19^{\circ}$. Thirty-two direction diffusion weighted images were acquired using the following sequence: repetition time/echo time/inversion time $=8400 / 68 / 90 \mathrm{~ms}$, b-value $=1000 \mathrm{~s} / \mathrm{mm}^{2}, 55$ slices, end resolution: $2.5 \times 2.5 \times 2.5 \mathrm{~mm}$, field of view: $240 \mathrm{~mm} \times 240 \mathrm{~mm}, 96 \times 96$ matrix, repeated twice. All scans were examined by a radiologist to rule out for structural abnormalities; none were reported for control participants. Prior to analyses, all participant scans were visually inspected for significant head movements and artifacts.

\section{Study protocol approval and patient consent}

This study was approved by the South Eastern Sydney Local Health District and the University of New South Wales human ethics committees, and carried out in accordance with the declaration of
Helsinki. Written informed consent was obtained prior to cognitive assessment and MRI scanning from the participant and/or primary carer.

\section{Data analysis}

Behavioral data were analyzed using IBM SPSS statistics (version 21.0; IBM Corp., Armonk, NY). Kolmogorov-Smirnov tests were run to determine the suitability of variables for parametric analyses. Analyses of variance (ANOVA), followed by Tukey post-hoc tests, were used to explore main effects between participant groups on age and education demographic variables. Independent samples $t$-test were used to analyze disease duration and the FRS between lvPPA and svPPA patient groups. ACE-R performance was analyzed using Kruskal-Wallis tests, followed by post-hoc Mann-Whitney tests. SYD-BAT performance was analyzed using Mann-Whitney tests. Progressive language and speech impairment performance at follow-up, compared to baseline, on the SYDBAT was analyzed using one-tailed Wilcoxon tests. In all analyses $p$ values $<0.05$ were considered to be significant. 


\section{Imaging analysis}

Differences in whole-brain fractional anisotropy (FA) were examined for mean group changes at baseline and follow-up, and longitudinally for interindividual change across time. TBSS [17] was used to perform a skeleton-based analysis of white matter FA, complemented by DTI-TK to provide a tensorbased registration for analyses [16]. Tensor based registration of diffusion weighted data is superior to traditional scalar based registration methods in constructing an unbiased spatially normalized brain atlas [18], with good reproducibility of diffusion metrics [19]. Serial diffusion-weighted sequences from each participant were averaged to improve signal-to-noise ratio before being corrected for eddy current distortions. A binary brain mask was generated [20] from the non-diffusion volume $\left(\mathrm{b}_{0}\right)$ and used to fit a tensor model onto diffusion-weighted images [21]. A wholebrain diffusion tensor image from each participant was used to generate study-specific brain atlases in DTI-TK. Group atlases were constructed through an iterative process of initial rigid registration, followed by non-linear registration, to determine the best mapping with each individual's scan using the least amount of deformation [16, 19]. For the longitudinal analysis, each individual's initial and follow-up scan was co-registered to generate a within-subject template representing a halfway transformation between the two timepoints, prior to construction of a group atlas [19]. Group atlases were registered to the Illinois Institute of Technology standard brain atlas, version 4.0 [22]. Transformation matrices were generated for each stage of registration to create a deformation field that defines the mapping directly from each individual's native scan space to standard space in a single interpolation [19]. Individual FA maps are then generated, concatenated, and skeletonized [17] to define the lines of maximum FA, which correspond to the centers of white matter tracts. Group FA skeletons were tested for significant differences using voxel-wise general linear modeling via permutation-based non-parametric testing [23] with 5000 permutations per contrast. Axial and radial diffusivity were also assessed in the same manner. Age was included as a nuisance variable in these analyses. Reported clusters were threshold-free cluster enhancement (TFCE) corrected for multiple comparisons at $p<0.05$. Delineation of white matter tracts implicated in significant clusters was performed with reference to Johns Hopkins University tractography atlas. Anatomically specific masks of the uncinate fasciculus (UF), inferior longitudinal fasciculus (ILF), and superior longitudinal fasciculus (SLF) were generated from the atlas using a threshold of $30 \%$, and visually inspected to ensure appropriate coverage. Using these masks, DTI metrics were extracted across both hemispheres, at baseline and follow-up, for atlas-based voxel-wise region of interest (ROI) analyses in patient and control cohorts, using repeated measures ANOVA in SPSS.

\section{RESULTS}

\section{Demographics and cognitive testing}

PPA patient groups were well matched for age, education, disease duration, and functional severity (FRS) (Table $1 ; p$ values $>0.87$ ). The control cohort was older than both patients groups (both $p$ values $<0.05$ ). Screening of general cognition (ACE-R: Total) indicated significant group differences $(p<0.001)$ with controls performing the highest. PPA patient groups did not show a significant difference in overall cognitive performance at baseline $(p=0.51)$ or 1 -year follow-up $(p=0.14)$, but did show a significant decline over a 1-year period ( $p$ values $<0.02$ ). Control participants did not show a decline in general cognition in the same period $(p=0.37)$.

PPA patient groups showed dissociable patterns of performance on language specific tasks, namely, lvPPA scored higher than svPPA on single-word comprehension, naming, and semantic association, but not repetition (Table 1). Overall pattern of impairment for single-word language tasks and performance on the SYD-BAT is consistent with the literature, and all patients performed 2 standard deviations below normative control performance, except for svPPA on single-word repetition [15]. At baseline, significant differences in language impairment was observed for naming, repetition, and semantic association ( $p$ values $<0.05)$, but not comprehension $(p=0.13)$. At followup, however, significant differences were found for comprehension, as well as repetition and semantic association ( $p$ values $<0.05$ ), but not naming $(p=0.14$ ). This change can be explained by the distinct pattern of progressive impairment on the SYD-BAT subscales between lvPPA and svPPA patients over the 1-year period. PPA patient groups showed dissociable pattern of longitudinal change on SYD-BAT subscales, with a significant decline in repetition for lvPPA $(p<0.001)$, while comprehension and semantic association both declined in svPPA ( $p$ values <0.05). In contrast, naming performance on the SYD-BAT worsened significantly in both patient groups over the 1 -year period ( $p$ values $<0.05)$. 


\section{Cross-sectional white matter integrity}

Whole-brain white matter integrity was compared at baseline between PPA patient groups and the control cohort (Fig. 1). Patient groups showed dissociation in the localization of white matter abnormality. At baseline, significant focal FA reduction was observed in the posterior region of the left ILF in lvPPA patients. SvPPA patients also showed focal left hemispheric white matter degradation involving the UF and anterior region of the ILF. Progressive deterioration along the length of the left ILF in PPA patient groups, from focal baseline regions of change, is highlighted using the same cross-sectional analysis approach at 1-year follow-up in Supplementary Figure 1. Axial and radial diffusivity metrics did not produce statistically significant clusters.

\section{Longitudinal white matter integrity}

Longitudinal inter-individual change in wholebrain white matter integrity was examined within each participant group (Fig. 2). Control participants did not show any significant change in FA over a 1-year followup period. LvPPA patients showed predominantly left hemispheric white matter reduction in FA, involving the UF, ILF, and temporal portion of the SLF. The same pattern of change was detected, albeit to a lesser extent, in the right hemisphere. In addition, significant clusters were detected in the left anterior thalamic radiation and genu of the corpus callosum. SvPPA patients showed the same pattern of focal left hemispheric white matter degradation observed in the cross-sectional analysis, with involvement primarily of the anterior portion of the ILF, and UF. Longitudinal inter-individual comparisons, however, also detected significant clusters of change in the posterior portion of the left ILF and right UF. Similar to lvPPA, significant clusters were also detected in the left anterior thalamic radiation and genu of the corpus callosum in svPPA. A direct contrast of longitudinal inter-individual change between PPA cohorts indicated significantly greater degradation in white matter integrity of the left ILF and SLF in lvPPA, compared to svPPA (Supplementary Figure 2).

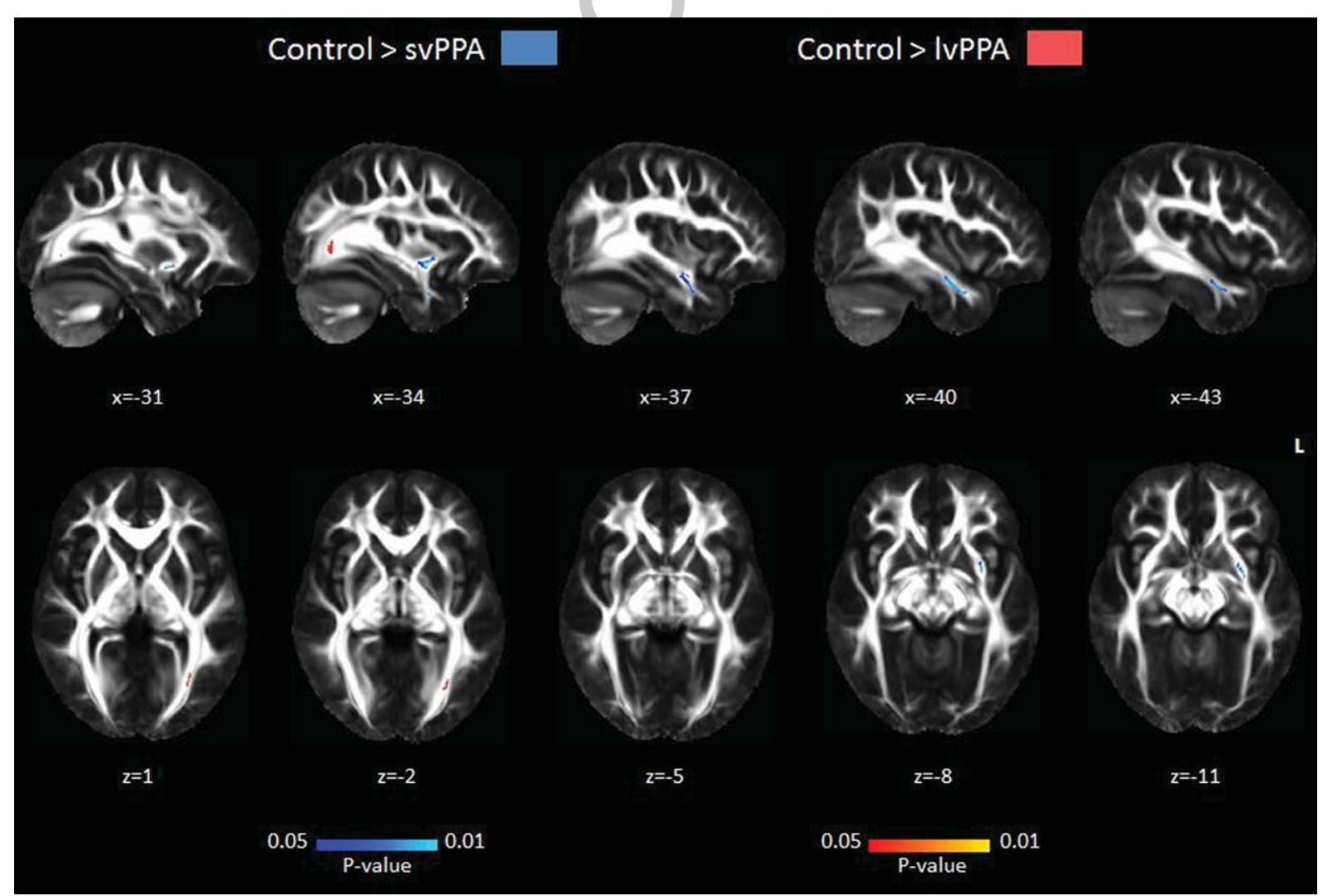

Fig. 1. Mean cross-sectional white matter tract degeneration in PPA patients at baseline compared to healthy controls. SvPPA showed reduced FA in the left UF and anterior region of the ILF. LvPPA showed reduced FA in the left posterior region of the ILF. Clusters were corrected for multiple comparisons and significant at $p<0.05$. Co-ordinates are provided in MNI standard space. 


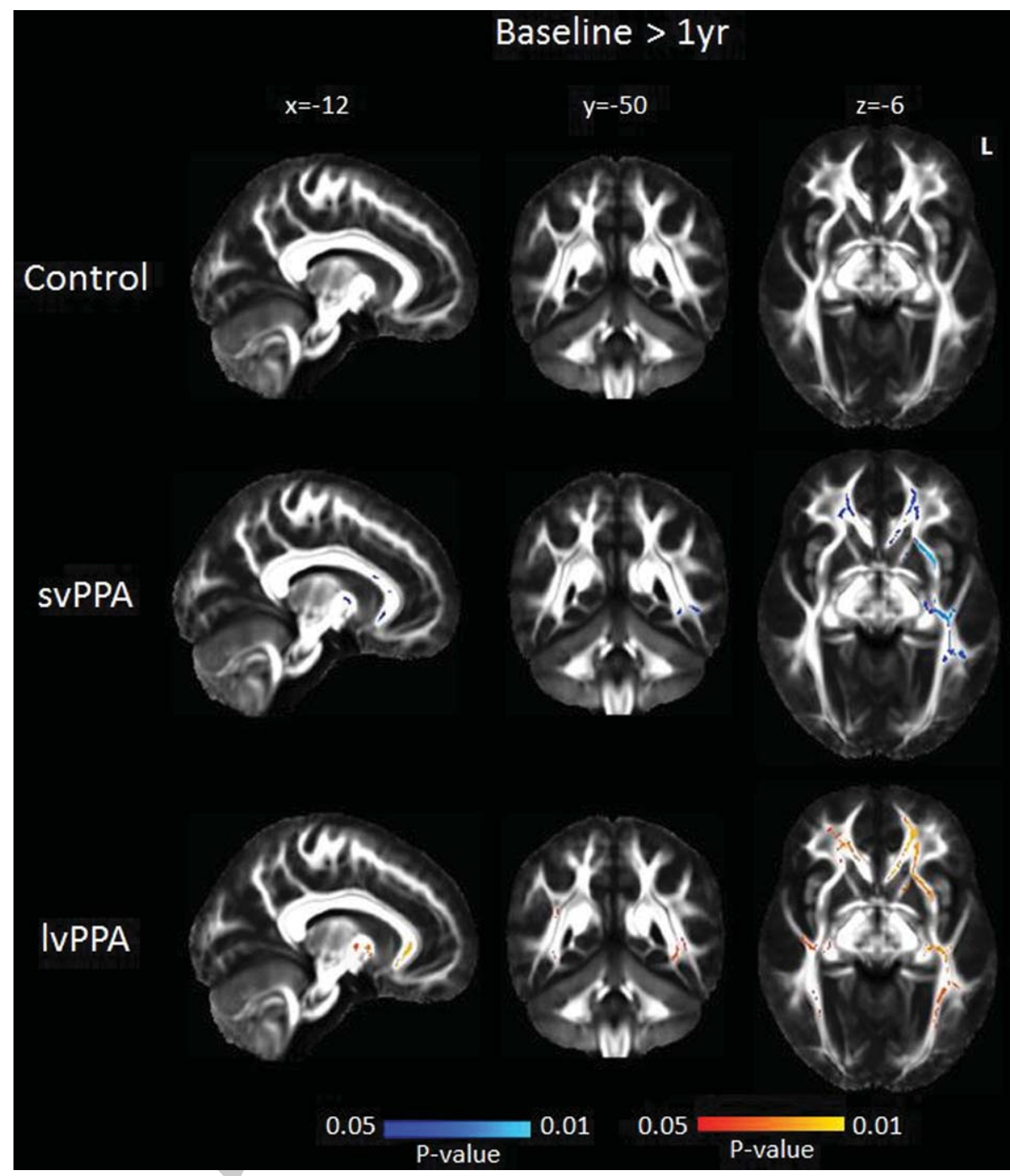

Fig. 2. Mean inter-individual white matter tract degeneration in PPA patients and healthy controls after 1 year. Control showed no significant FA reduction. SvPPA showed reduced FA in the left ILF, SLF, and bilateral UF. LvPPA showed reduced FA in the UF, ILF, and SLF bilaterally. Clusters are corrected for multiple comparisons and significant at $p<0.05$. Co-ordinates are provided in MNI standard space.

This pattern is consistent with the longitudinal within group analyses.

ROI analyses examining FA in the UF, ILF, and SLF tracts were carried out using repeated measures ANOVA, comparing tracts, and time, separately for left and right hemispheres across groups. Significant differences were found bilaterally across groups across tracts (all $p$ values $<0.001$ ) and time (all $p$ values $<0.02)$. A significant interaction effect was found between group, tract and time for the right hemisphere $[\mathrm{F}(4,72)=4.15, p=0.004]$, but not left hemisphere $[F(4,72)=2.13, p=0.085]$. Post-hoc contrasts of the right UF, ILF, and SLF did not significantly differ from controls at baseline or follow-up in PPA patient groups (all $p$ values $>0.2$ ). This may be attributed to obtaining a mean FA value for the entire length of these tracts, reducing statistical power. LvPPA patients, however, showed significantly reduced FA across time in the right UF $(p=0.01)$, and a trend toward significance in the right ILF $(p=0.07)$ and SLF ( $p=0.06)$ (Table 2). SvPPA patients showed significantly reduced FA across time only in the right UF $(p=0.01)$. In the left hemisphere, significant differences were observed in the left UF and ILF for svPPA patients at baseline and follow-up (all $p$ values $<0.01$ ), compared to controls. In contrast, lvPPA 
Table 2

Fractional anisotropy of the uncinate fasciculus, inferior longitudinal fasciculus, and superior longitudinal fasciculus tracts in healthy controls and PPA groups, at baseline and 1 year. Mean and standard deviation reported

\begin{tabular}{lccc}
\hline Baseline & \multicolumn{3}{c}{ FA } \\
\cline { 2 - 4 } & UF & ILF & SLF \\
\hline Control & & & \\
$\quad$ Left Hemisphere & $0.39(0.03)$ & $0.42(0.05)$ & $0.44(0.03)$ \\
Right Hemisphere & $0.42(0.04)$ & $0.5(0.04)$ & $0.45(0.03)$ \\
svPPA & & & \\
Left Hemisphere & $0.31(0.05)^{\mathrm{a}}$ & $0.37(0.04)^{\mathrm{a}}$ & $0.44(0.03)$ \\
$\quad$ Right Hemisphere & $0.43(0.05)$ & $0.51(0.03)$ & $0.46(0.04)$ \\
lvPPA & & & \\
Left Hemisphere & $0.39(0.04)$ & $0.4(0.03)$ & $0.42(0.03)$ \\
Right Hemisphere & $0.43(0.05)$ & $0.49(0.04)$ & $0.46(0.03)$ \\
\hline 1 Year & UF & ILF & SLF \\
\hline Control & & & \\
Left Hemisphere & $0.39(0.03)$ & $0.43(0.04)$ & $0.44(0.03)$ \\
Right Hemisphere & $0.42(0.03)$ & $0.5(0.04)$ & $0.46(0.03)$ \\
svPPA & & & \\
$\quad$ Left Hemisphere & $0.28(0.05)^{\mathrm{a}, \mathrm{b}}$ & $0.36(0.04)^{\mathrm{a}, \mathrm{b}}$ & $0.43(0.04)^{\mathrm{b}}$ \\
Right Hemisphere & $0.4(0.06)^{\mathrm{b}}$ & $0.51(0.05)$ & $0.46(0.04)$ \\
lvPPA & & & \\
$\quad$ Left Hemisphere & $0.38(0.04)^{\mathrm{b}}$ & $0.38(0.03)^{\mathrm{a}, \mathrm{b}}$ & $0.42(0.03)$ \\
Right Hemisphere & $0.41(0.04)^{\mathrm{b}}$ & $0.48(0.04)$ & $0.45(0.03)$ \\
\hline
\end{tabular}

UF, uncinate fasciculus; ILF, inferior longitudinal fasciculus; SLF, superior longitudinal fasciculus. Significant at $p<0.05$; ${ }^{\mathrm{a}}$ significant change compared to control; ${ }^{b}$ significant change from baseline.

patients only showed significant differences in the left ILF at follow-up ( $p=0.03)$, compared to controls. Across time, svPPA showed significantly reduced FA in the left UF, ILF, and SLF (all $p$-values <0.01), while lvPPA showed significant reduction in the left UF and ILF (all $p$-values <0.05).

\section{DISCUSSION}

This study examined longitudinal white matter integrity in lvPPA and svPPA with a focus on the pattern of change over a 1-year period. We uncovered different patterns in the degree of disease propagation in these patient groups, with lvPPA showing relatively diffuse white matter changes, compared to the focal changes observed in svPPA. Both PPA patient groups showed deterioration predominantly in the left hemispheric UF, ILF, and SLF, with additional involvement of their homologous tracts in the contralateral hemisphere. Clinically, the lvPPA and svPPA cohorts were well defined showing clear progressive decline on cognitive tasks and dissociation in degree of impaired speech production and comprehension, respectively. The divergent course of white matter degeneration in lvPPA and svPPA cohorts highlight the possibility of utilizing advanced longitudinal neuroimaging to better clarify underlying pathology in vivo.

Degradation of the left UF and ILF has consistently been implicated as the main white matter tracts affected across the PPA spectrum [11, 24], with some evidence of SLF involvement [25, 26]. These tracts have also been identified as being critical components of the language network [12, 25, 27], in particular speech production and semantic retrieval [27, 28]. A recent cross-sectional study dissociated patterns of white matter change in lvPPA and svPPA [11]. Specifically, lvPPA demonstrate widespread left lateralized involvement of the UF and ILF while in SvPPA white matter change is predominantly found in ventral tracts, involving bilateral UF and left ILF. Findings from the current study are consistent with the signature of white matter change in svPPA $[11,26]$. Longitudinal imaging, however, revealed a complex pattern of progressive white matter degradation in lvPPA, in particular, dissociating from svPPA. Cross-sectional analyses at baseline demonstrated that early white matter pathology in lvPPA tend to be circumscribed to the posterior tail of the left ILF. Over a 12-month period, however, our analyses showed that changes became widespread, affecting the entire length of the left ILF and involving the UF and SLF in this group. These analyses further demonstrated a similar pattern of white matter changes in the right hemisphere, albeit to a lesser extent. These distinct differences in localization and laterality of white matter abnormality allude to divergent underlying pathology and their propagation in the logopenic and semantic variants of PPA.

The focal propagation of white matter dysfunction in svPPA followed a stereotypical pattern along anatomical tracts, suggesting that pathology spreads throughout neighboring axonal tracts [29]. In contrast, lvPPA showed a diffuse white matter involvement displaying a less stereotyped pattern of progression that not necessarily follows the trajectory of axonal bundles. This divergent pattern of white matter dysfunction mirrors extensive structural imaging and pathological evidence that shows widespread brain atrophy in lvPPA and circumscribed atrophy in svPPA [3, 30], and suggests fundamental mechanistic differences in how pathology spreads. Whereas the primary event driving white matter involvement in svPPA is axonal death (i.e., Wallerian degeneration) derived from focal neuronal death [29], the pattern of white matter involvement in lvPPA seems to be different. Our findings suggest a combined spreading characterized by eccentric white matter changes over time associated with distant multi-centric involvement. This 
pattern suggests that pathology spreads in two different fashions: directly from neuron to neuron throughout axonal bundles, as well as multiple distant pathological seeds that do not necessarily follow anatomical connections. This pattern highlights the central role of extra-neuronal factors in lvPPA, in particular, the neurotoxic effect of amyloid- $\beta$. Interestingly, white matter dysfunction was also detected in the genu of the corpus callosum, perhaps presenting an avenue for transcallosal propagation. Further research is needed to investigate the differential regional effect of amyloid and neuronal vulnerability to clarify this issue.

White matter change is increasingly being used to address one of the prevalent issues in the field that remains unresolved from decades of structural imaging, which is the accurate prediction of pathology in vivo. Although important advances in the development of biomarkers have taken place in recent years, clinicopathological correlations at an individual level remain elusive. This issue is particularly acute in PPA cases due to $\mathrm{AD}$, a pervasive pathology that can adopt varied neurocognitive patterns and is often present in unclassified PPA cases due to mixed deficits [31]. In this sense, white matter changes over time can provide a complementary account that can assist in the identification of AD cases. Current findings suggest the posterior tail of the left ILF is a sensitive marker of white matter change in the early stages of the disease.

In the current study, efforts were made in the careful selection of well-defined PPA cases fulfilling diagnostic criteria [1] with the aid of experienced neurologists. A limitation is, however, the lack of genetic testing and pathological confirmation of underlying disease pathology. PiB PET imaging was included as a proxy measure of $\mathrm{AD}$ pathology and was available for the majority of lvPPA patients, but only half of svPPA patients. For PPA patients with available PiB imaging, however, all lvPPA were found to be PiB-positive while svPPA were PiB-negative, consistent with expected pathology [8] and interpretation of current imaging findings. All patient and control follow-up assessments occurred within a 2-month margin of the 1-year timepoint, representing genuine longitudinal cognitive and neural change. Robust longitudinal imaging analyses were carried out using tensor-based registration for accurate registration of tract orientation [16] and localization of white matter changes. Current findings should, however, be replicated in a larger sample of PPA patients for reliability.

In conclusion, logopenic and semantic variants of PPA demonstrate marked behavioral and neural profiles resulting in progressive deterioration of distinct neural networks. Modeling the progression of white matter degradation along tracts provides new insight into the molecular mechanisms underlying disease pathology.

\section{ACKNOWLEDGMENTS}

This work was supported by funding to Forefront, a collaborative research group dedicated to the study of frontotemporal dementia and motor neuron disease, from the National Health and Medical research Council (NHMRC) of Australia program grant (\#1037746), the Australian Research Council (ARC) Centre of Excellence in Cognition and its Disorders Memory Node (\#CE110001021) and an ARC Discovery Project grant (DP1093279). ST is supported by Alzheimer's Australia Dementia Research Foundation and NHMRC of Australia awards. CEL is supported by DVC post-doctoral fellowship of the University of Sydney (S0716/U2644). OP is supported by a NHMRC Career Development Fellowship (APP1022684). MH is supported by Alzheimer Research UK and the Isaac Newton Trust. These funding sources had no involvement in the study design, collection, analysis and interpretation of data, writing the manuscript, and in the decision to submit the manuscript for publication. We are grateful to the research participants and carers involved with ForeFront research studies.

Authors' disclosures available online (http://j-alz. com/manuscript-disclosures/15-0626r1).

\section{SUPPLEMENTARY MATERIAL}

The supplementary material is available in the electronic version of this article: http://dx.doi.org/10.3233/ JAD-150626.

\section{REFERENCES}

[1] Gorno-Tempini ML, Hillis AE, Weintraub S, Kertesz A, Mendez M, Cappa SF, Ogar JM, Rohrer JD, Black S, Boeve BF, Manes F, Dronkers NF, Vandenberghe R, Rascovsky K, Patterson K, Miller BL, Knopman DS, Hodges JR, Mesulam MM, Grossman M (2011) Classification of primary progressive aphasia and its variants. Neurology 76, 1006-1014.

[2] Gorno-Tempini ML, Dronkers NF, Rankin KP, Ogar JM, Phengrasamy L, Rosen HJ, Johnson JK, Weiner MW, Miller BL (2004) Cognition and anatomy in three variants of primary progressive aphasia. Ann Neurol 55, 335-346.

[3] Rohrer JD, Ridgway GR, Crutch SJ, Hailstone J, Goll JC, Clarkson MJ, Mead S, Beck J, Mummery C, Ourselin S, Warrington EK, Rossor MN, Warren JD (2010) Progressive 
logopenic/phonological aphasia: Erosion of the language network. Neuroimage 49, 984-993.

[4] Rohrer JD, Caso F, Mahoney C, Henry M, Rosen HJ, Rabinovici G, Rossor MN, Miller B, Warren JD, Fox NC, Ridgway GR, Gorno-Tempini ML (2013) Patterns of longitudinal brain atrophy in the logopenic variant of primary progressive aphasia. Brain Lang 127, 121-126.

[5] Heim S, Pieperhoff P, Grande M, Kuijsten W, Wellner B, Saez LE, Schulte S, Sudmeyer M, Caspers S, Minnerop M, Binkofski F, Huber W, Amunts K (2014) Longitudinal changes in brains of patients with fluent primary progressive aphasia. Brain Lang 131, 11-19.

[6] Brambati SM, Amici S, Racine CA, Neuhaus J, Miller Z, Ogar J, Dronkers N, Miller BL, Rosen H, Gorno-Tempini ML (2015) Longitudinal gray matter contraction in three variants of primary progressive aphasia: A tenser-based morphometry study. Neuroimage Clin 8, 345-355.

[7] Mackenzie IR, Neumann M, Bigio EH, Cairns NJ, Alafuzoff I, Kril J, Kovacs GG, Ghetti B, Halliday G, Holm IE, Ince PG, Kamphorst W, Revesz T, Rozemuller AJ, Kumar-Singh S, Akiyama H, Baborie A, Spina S, Dickson DW, Trojanowski JQ, Mann DM (2010) Nomenclature and nosology for neuropathologic subtypes of frontotemporal lobar degeneration: An update. Acta Neuropathol 119, 1-4.

[8] Chare L, Hodges JR, Leyton CE, McGinley C, Tan RH, Kril JJ, Halliday GM (2014) New criteria for frontotemporal dementia syndromes: Clinical and pathological diagnostic implications. J Neurol Neurosurg Psychiatry 85, 865-870.

[9] Mesulam M, Wicklund A, Johnson N, Rogalski E, Leger GC, Rademaker A, Weintraub S, Bigio EH (2008) Alzheimer and frontotemporal pathology in subsets of primary progressive aphasia. Ann Neurol 63, 709-719.

[10] Deramecourt V, Lebert F, Debachy B, Mackowiak-Cordoliani MA, Bombois S, Kerdraon O, Buee L, Maurage CA, Pasquier F (2010) Prediction of pathology in primary progressive language and speech disorders. Neurology 74, 42-49.

[11] Mahoney CJ, Malone IB, Ridgway GR, Buckley AH, Downey LE, Golden HL, Ryan NS, Ourselin S, Schott JM, Rossor MN, Fox NC, Warren JD (2013) White matter tract signatures of the progressive aphasias. Neurobiol Aging 34, 1687-1699.

[12] Powers JP, McMillan CT, Brun CC, Yushkevich PA, Zhang H, Gee JC, Grossman M (2013) White matter disease correlates with lexical retrieval deficits in primary progressive aphasia. Front Neurol 4, 212.

[13] Mioshi E, Hsieh S, Savage S, Hornberger M, Hodges JR (2010) Clinical staging and disease progression in frontotemporal dementia. Neurology 74, 1591-1597.

[14] Mioshi E, Dawson K, Mitchell J, Arnold R, Hodges JR (2006) The Addenbrooke's Cognitive Examination Revised (ACE-R): A brief cognitive test battery for dementia screening. Int J Geriatr Psychiatry 21, 1078-1085.

[15] Savage S, Hsieh S, Leslie F, Foxe D, Piguet O, Hodges JR (2013) Distinguishing subtypes in primary progressive aphasia: Application of the Sydney language battery. Dement Geriatr Cogn Disord 35, 208-218.

[16] Zhang H, Avants BB, Yushkevich PA, Woo JH, Sumei Wang, McCluskey LF, Elman LB, Melhem ER, Gee JC (2007) High-dimensional spatial normalization of diffusion tensor images improves the detection of white matter differences:
An example study using amyotrophic lateral sclerosis. IEEE Trans Med Imaging 26, 1585-1597.

[17] Smith SM, Jenkinson M, Johansen-Berg H, Rueckert D, Nichols TE, Mackay CE, Watkins KE, Ciccarelli O, Cader MZ, Matthews PM, Behrens TE (2006) Tract-based spatial statistics: Voxelwise analysis of multi-subject diffusion data. Neuroimage 31, 1487-1505.

[18] Bach M, Laun FB, Leemans A, Tax CM, Biessels GJ, Stieltjes B, Maier-Hein KH (2014) Methodological considerations on tract-based spatial statistics (TBSS). Neuroimage 100, 358-369.

[19] Keihaninejad S, Zhang H, Ryan NS, Malone IB, Modat M, Cardoso MJ, Cash DM, Fox NC, Ourselin S (2013) An unbiased longitudinal analysis framework for tracking white matter changes using diffusion tensor imaging with application to Alzheimer's disease. Neuroimage 72, 153-163.

[20] Smith SM (2002) Fast robust automated brain extraction. Hum Brain Mapp 17, 143-155.

[21] Smith SM, Jenkinson M, Woolrich MW, Beckmann CF, Behrens TE, Johansen-Berg H, Bannister PR, De Luca M, Drobnjak I, Flitney DE, Niazy RK, Saunders J, Vickers J, Zhang Y, De Stefano N, Brady JM, Matthews PM (2004) Advances in functional and structural MR image analysis and implementation as FSL. Neuroimage 23(Suppl 1), S208-S219.

[22] Varentsova A, Zhang S, Arfanakis K (2014) Development of a high angular resolution diffusion imaging human brain template. Neuroimage 91, 177-186.

[23] Nichols TE, Holmes AP (2002) Nonparametric permutation tests for functional neuroimaging: A primer with examples. Hum Brain Mapp 15, 1-25.

[24] Agosta F, Scola E, Canu E, Marcone A, Magnani G, Sarro L, Copetti M, Caso F, Cerami C, Comi G, Cappa SF, Falini A, Filippi M (2012) White matter damage in frontotemporal lobar degeneration spectrum. Cereb Cortex 22, 2705-2714.

[25] Galantucci S, Tartaglia MC, Wilson SM, Henry ML, Filippi M, Agosta F, Dronkers NF, Henry RG, Ogar JM, Miller BL, Gorno-Tempini ML (2011) White matter damage in primary progressive aphasias: A diffusion tensor tractography study. Brain 134, 3011-3029.

[26] Lam BY, Halliday GM, Irish M, Hodges JR, Piguet O (2014) Longitudinal white matter changes in frontotemporal dementia subtypes. Hum Brain Mapp 35, 3547-3557.

[27] Saur D, Kreher BW, Schnell S, Kummerer D, Kellmeyer P, Vry MS, Umarova R, Musso M, Glauche V, Abel S, Huber W, Rijntjes M, Hennig J, Weiller C (2008) Ventral and dorsal pathways for language. Proc Natl Acad Sci U S A 105, 18035-18040.

[28] Hickok G, Poeppel D (2007) The cortical organization of speech processing. Nat Rev Neurosci 8, 393-402.

[29] Fletcher PD, Warren JD (2011) Semantic dementia: A specific network-opathy. J Mol Neurosci 45, 629-636.

[30] Wicklund MR, Duffy JR, Strand EA, Machulda MM, Whitwell JL, Josephs KA (2014) Quantitative application of the primary progressive aphasia consensus criteria. Neurology 82, 1119-1126.

[31] Mesulam MM, Wieneke C, Thompson C, Rogalski E, Weintraub S (2012) Quantitative classification of primary progressive aphasia at early and mild impairment stages. Brain 135, 1537-1553. 